This is a preprint of: "Piecewise linear differential systems with two real saddles", Joan Carles Artés, Jaume Llibre, Joao Carlos Medrado, Marco Antonio Teixeira, Math. Comput. Simulation, vol. 95, 13-22, 2013.

\title{
Piecewise linear differential systems with two real saddles
}

\author{
Joan C. Artés ${ }^{\mathrm{a}}$, Jaume Llibre ${ }^{\mathrm{a}}$, João C. Medrado ${ }^{\mathrm{b}}$, Marco A. Teixeira ${ }^{\mathrm{c}, *}$ \\ ${ }^{a}$ Departament de Matemàtiques, Universitat Autònoma de Barcelona, 08193 Bellaterra, Barcelona, \\ Catalonia, Spain. \\ ${ }^{b}$ Instituto de Matemática e Estatística, Universidade Federal de Goiás, 74001-970 Goiânia, Goiás, Brazil. \\ ${ }^{c}$ Departamento de Matemática, Universidade Estadual de Campinas, Caixa Postal 6065, 13083-970, \\ Campinas, SP, Brazil.
}

\begin{abstract}
In this paper we study piecewise linear differential systems formed by two regions separated by a straight line so that each system has a real saddle point in its region of definition. If both saddles are conveniently situated, they produce a transition flow from a segment of the splitting line to another segment of the same line, and this produces a generalized singular point on the line. This point is a focus or a center and there can be found limit cycles around it. We are going to show that the maximum number of limit cycles that can bifurcate from this focus is two. One of them appears through a Hopf bifurcation and the second when the focus becomes a node by means of the sliding.
\end{abstract}

Keywords: non-smooth differential system, limit cycle, piecewise linear differential system, Hopf bifurcation, sliding limit cycle.

2000 MSC: 34C05, 34C07, 37G15.

\section{Introduction}

One of the main problems in the qualitative theory of real planar differential systems is the determination of limit cycles. Limit cycles of planar differential systems were defined by Poincaré [18]. At the end of the 1920s van der Pol [19], Liénard [16] and Andronov [1] proved that a closed orbit of a self-sustained oscillation occurring in a vacuum tube circuit was a limit cycle as considered by Poincaré. After these works, the non-existence, existence, uniqueness and other properties of limit cycles were studied extensively by mathematicians and physicists, and more recently also by chemists, biologists, economists, etc. (see for 
instance the books $[5,20])$. In this paper we are interested in studying the limit cycles of a class of non-smooth differential systems.

A large number of problems from mechanics, electrical engineering and the theory of automatic control are described by non-smooth differential systems, see [2]. The basic methods of qualitative theory for this kind of differential systems were established or developed in the book [9] and in a large number of papers, see for instance [3, 4, 7, 11, 10, 12, 13]. Also the problem of Hopf bifurcation in some of these problems have been studied in $[6,15,17,21]$. Besides planar linear global differential systems can have at most 10 (7 non degenerated) different phase portraits (see for instance [8]) and no limit cycles, the number of different phase portraits, and the maximum number of limit cycles of piecewise linear systems (even with as few as two regions) is still unknown. The fact that we can situate on each region a linear system with either a real singular point, or a virtual one, plus the fact that another singular point may appear on the splitting line by the combination of the two flows, and the possible existence of limit cycles, increases a lot the number of possible phase portraits of two piecewise linear differential systems.

Consider a planar differential system of the form

$$
\dot{x}=f(x, y), \quad \dot{y}=g(x, y), \quad x \neq 0
$$

where

$$
(f(x, y), g(x, y))= \begin{cases}\left(f^{+}(x, y), g^{+}(x, y)\right), & x>0 \\ \left(f^{-}(x, y), g^{-}(x, y)\right), & x<0\end{cases}
$$

and $f^{ \pm}$and $g^{ \pm}$are linear functions on $\mathbb{R}^{2}$. This defines the following two linear differential systems

$$
\left(\begin{array}{l}
\dot{x} \\
\dot{y}
\end{array}\right)=\left(\begin{array}{ll}
a^{+} & b^{+} \\
c^{+} & d^{+}
\end{array}\right)\left(\begin{array}{l}
x-\alpha^{+} \\
y-\beta^{+}
\end{array}\right)=\left(\begin{array}{l}
f^{+}(x, y) \\
g^{+}(x, y)
\end{array}\right)
$$

and

$$
\left(\begin{array}{l}
\dot{x} \\
\dot{y}
\end{array}\right)=\left(\begin{array}{ll}
a^{-} & b^{-} \\
c^{-} & d^{-}
\end{array}\right)\left(\begin{array}{l}
x-\alpha^{-} \\
y-\beta^{-}
\end{array}\right)=\left(\begin{array}{l}
f^{-}(x, y) \\
g^{-}(x, y)
\end{array}\right) .
$$

We call (2) and (3) the right and the left subsystem of (1) respectively. From $[4,9,12]$ we know that the flow of $(1)$ denoted by $\varphi(t, A)$ can be defined by using the flows $\varphi^{ \pm}(t, A)$ of (2) and (3). For example, for a point $A \in \mathbb{R}_{+}^{2} \cup \mathbb{R}_{-}^{2}$ where $\mathbb{R}_{ \pm}^{2}=\{(x, y): \pm x>0\}$, we have

$$
\varphi(t, A)=\varphi^{ \pm}(t, A) \quad \text { if } \quad \varphi^{ \pm}(t, A) \in \mathbb{R}_{ \pm}^{2} .
$$

We will call $(x, y)$ a real (respectively virtual) singular point of (2) if it is a singular point of $(2)$ with $x>0$ (respectively $x<0$ ). An equivalent definition can be done for $(3)$.

For a point $A \notin \mathbb{R}_{+}^{2} \cup \mathbb{R}_{-}^{2}$ satisfying $f^{+}(A) f^{-}(A)>0$ we define $\varphi(t, A)$ as follows

$$
\varphi(t, A)= \begin{cases}\varphi^{-}(t, A), & t<0 \text { and } \varphi^{-}(t, A) \in \mathbb{R}_{-}^{2}, \\ A, & t=0, \\ \varphi^{+}(t, A), & t>0 \text { and } \varphi^{+}(t, A) \in \mathbb{R}_{+}^{2},\end{cases}
$$


if $f^{+}(A)>0, f^{-}(A)>0$; and

$$
\varphi(t, A)= \begin{cases}\varphi^{+}(t, A), & t<0 \text { and } \varphi^{+}(t, A) \in \mathbb{R}_{+}^{2} \\ A, & t=0, \\ \varphi^{-}(t, A), & t>0 \text { and } \varphi^{-}(t, A) \in \mathbb{R}_{-}^{2}\end{cases}
$$

if $f^{+}(A)<0, f^{-}(A)<0$.

Of course we have that $\varphi(t, A)=A$ for all $t$ if $A \in \mathbb{R}_{ \pm}^{2}$ is a singular point of the corresponding system (2) or (3). In this case $A$ is also called a singular point of (1).

Assume that $A \notin \mathbb{R}_{+}^{2} \cup \mathbb{R}_{-}^{2}$. If $f^{+}(A) f^{-}(A) \leq 0$ then the flow on $A$ is not defined and the point is called a generalized singular point of (1) and for the sake of completeness in this case we define $\varphi(t, A)=A$ for all $t$. When $f^{+}(A) f^{-}(A)<0$ by continuity, there is some interval of points on $x=0$ close to $A$ which are also generalized singular points, and then the flow on them can either be null or can move up or down depending of the slopes of both flows when arriving to $x=0$ producing the phenomena known as sliding. When $f^{+}(A)=f^{-}(A)=0$ and the multiplicity of the contact points of each flow with the line $x=0$ is even, and there is an interval on $x=0$ containing $A$ so that for each $B \neq A$ in that interval $f^{+}(B) f^{-}(B)>0$, then $A$ is an isolated generalized singular point, i.e. $A$ is not part of a segment of generalized singular points. All other cases of $f^{+}(A) f^{-}(A)=0$ produce generalized singular points which are part (possibly a border) of a segment of generalized singular points.

Since the study of linear differential systems is completely known from the works of Laplace in 1812, the only field of research in piecewise linear differential systems is about the orbits which move on both sides of the line $x=0$, and specially interesting is the possibility to have limit cycles surrounding a generalized singular point. In order to have such limit cycles the first thing that we need is an orbit which cuts transversally at least twice the line $x=0$. In other words that there exist a Poincaré map from $x=0$ to itself defined in $x \leq 0$, and another defined in $x \geq 0$. Every one of this Poincaré maps is of the form $\psi: I \rightarrow J$ with the (bounded or unbounded) intervals $I$ and $J$ of $x=0$ so that $\psi(I)=J$, then for $A \in I$ we have that $\varphi(t, A) \in J$ where the time $t$ may me different for each point of the interval $I$. The set of orbits going from the points of $I$ to the points of $J$ define what is called a transition flow.

In order to have closed orbits around a generalized singular point we must then have a transition flow $\psi^{+}: I^{+} \rightarrow J^{+}$and $\psi^{-}: I^{-} \rightarrow J^{-}$for each subsystem, so that the intersection of the respective segments $J^{+} \cap I^{-}$and $J^{-} \cap I^{+}$are not empty. There are many ways to produce such flows. The most studied one has been putting one virtual focus on each subsystem and then the whole line $x=0$ splits in three segments, the middle one formed by generalized singular points (can be a single point) such that the flow from one extreme segment goes to the other. It is also possible to have one virtual focus and one real, or even two real focus and produce transition flows, see for instance [15].

Another possible way to produce a transition flow (and less studied up to now) is having 


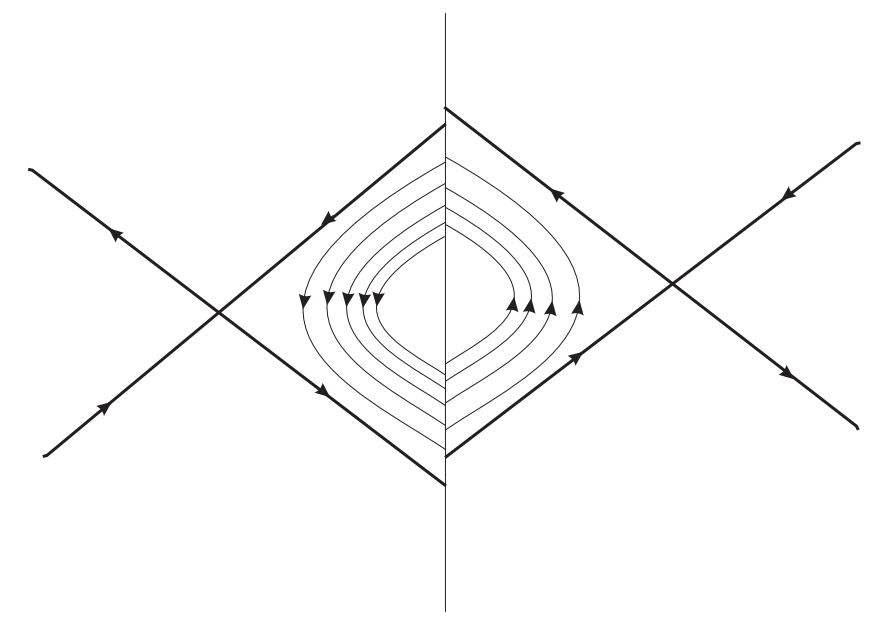

Figure 1: Piecewise linear differential system with two real saddles.

one real saddle on each subsystem so that no separatrix is parallel to $x=0$ and so that the bounded segments formed by the intersections of the two eigenvectors of each saddle with the line $x=0$ is not empty (see Figure 1 ). In this case we get a bounded transition flow. In order to study such a flow we are going to rewrite the flow as

$$
\left(\begin{array}{c}
\dot{x} \\
\dot{y}
\end{array}\right)=\left(\begin{array}{cc}
a^{+} & 1+b^{+} \\
1+c^{+} & d^{+}
\end{array}\right)\left(\begin{array}{c}
x-1-\alpha^{+} \\
y-\beta^{+}
\end{array}\right)=\left(\begin{array}{c}
f^{+}(x, y) \\
g^{+}(x, y)
\end{array}\right),
$$

and

$$
\left(\begin{array}{c}
\dot{x} \\
\dot{y}
\end{array}\right)=\left(\begin{array}{cc}
a^{-} & 1+b^{-} \\
1+c^{-} & d^{-}
\end{array}\right)\left(\begin{array}{c}
x+1-\alpha^{-} \\
y-\beta^{-}
\end{array}\right)=\left(\begin{array}{c}
f^{-}(x, y) \\
g^{-}(x, y)
\end{array}\right) .
$$

It is clear that equation (1) with subsystems (4) and (5) when $a^{ \pm}=b^{ \pm}=c^{ \pm}=$ $d^{ \pm}=\alpha^{ \pm}=\beta^{ \pm}=0$ has two real saddles at $(1,0)$ and $(-1,0)$, the respective eigenvectors cut $x=0$ at the points $(0,1)$ and $(0,-1)$ for which the flow holds $f^{+}(0,1) f^{-}(0,1)>0$ (same for $(0,-1))$ and so, two separatrices of one saddle and two from the other form a heteroclinic loop which encloses a bounded region (a square) in which interior we see branches of hiperbolas on each side and that by symmetry produce closed orbits. In short there is an isolated generalized singular point at $(0,0)$ which is a linear center (see Figure 2 ). Then for small perturbations of the twelve parameters so that we keep the existence of the two real saddles and a transition flow on each side which shares some segment, it is possible to define a Poincaré return map and determine the existence or not of limit cycles. So our main result is the following one.

Theorem 1. For any system (1) with subsystems (4) and (5) with infinitesimal parameters $0 \leq\left|a^{ \pm}\right|,\left|b^{ \pm}\right|,\left|c^{ \pm}\right|,\left|d^{ \pm}\right|,\left|\alpha^{ \pm}\right|,\left|\beta^{ \pm}\right|<<1$ so that it has two real saddles and the bounded segments formed by the intersections of the two eigenvectors of each saddle with the line $x=0$ is not empty and produces a transition flow, the maximum number of infinitesimal limit cycles that can be produced is exactly two. 


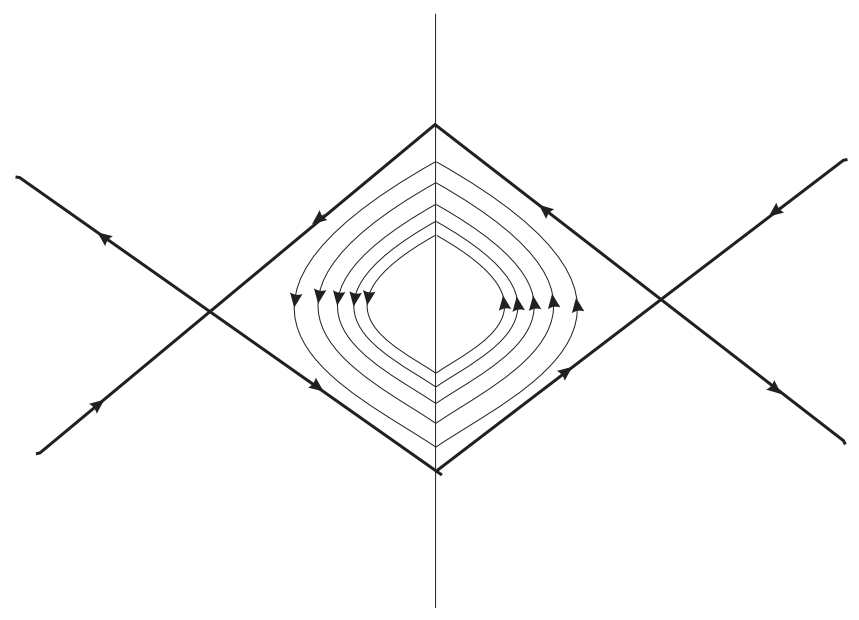

Figure 2: Piecewise linear differential system with two real saddles, and a center.

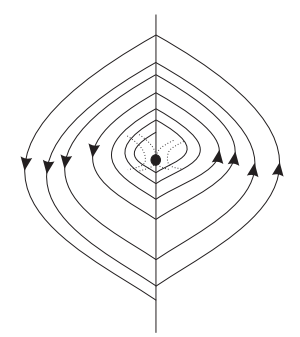

Figure 3: Isolated generalized singular point being a focus.

The paper is organized as follows. In section 2 we introduce some technical tools and in section 3 we prove Theorem 1.

Similar results to Theorem 1 were provided by Han and Zhang in [15], but for two piecewise linear differential systems with at least one focus in one piece, instead of a real saddle in each piece.

\section{Needed tools}

Whenever we have an isolated generalized singular point and a transition flow from points on one side of it to the other side which do not form a continuum of periodic orbits surrounding this singular point, this point is a focus and thus, it will be either stable or unstable (see Figure 3).

Such a system can always be infinitesimally perturbed in order to produce a segment of generalized singular points close to where we had before the isolated generalized singular point. That is we produce a segment with a sliding flow. Then one of these generalized 


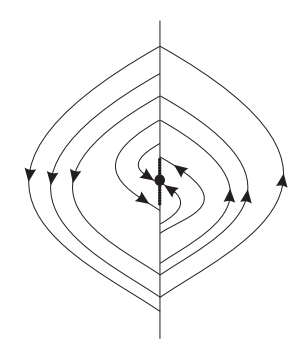

Figure 4: Sliding producing a sliding limit cycle.

singular points will be the limit point (either in positive or negative time) of two orbits (one on each side) with a definite slope. That is, one of the generalized singular points will be a stable or unstable node, and depending on the perturbation we can get either one or the other (see for instance Figure 4). So, since we can change the stability of the isolated generalized singular point, we can always produce a non-infinitesimal limit cycle around it playing with this type of perturbations. We call such a limit cycle a sliding limit cycle. As far as we know this kind of limit cycles where studied by first time in Kuznetsov, Rinaldi and Gragnani [14] where more details about them are provided (see also [15]).

In smooth polynomial systems one can always make affine changes of variables including a rescaling of the time which allows the reduction of 7 parameters. In piecewise differential systems formed by two regions separated by a straight line, we can do even more simplifications of the parameters. We can make two different affine changes, including different time rescalings on each subsystem keeping the following properties:

(i) the changes must be non degenerate,

(ii) each point of $(0, y)$ of $x=0$ must be transformed for both affine changes into the same $(0, z)$ again of $x=0$,

(iii) all the real and virtual singular points of each subsystem must remain on the same side as they were, and

(iv) the rescaling of the time must have the same sign on both subsystems.

All these conditions can be achieved by means of a twin change of variables like $(x, y, t) \rightarrow$ $(X, Y, T)$ given by

$$
\left(\begin{array}{l}
X \\
Y
\end{array}\right)=\left(\begin{array}{ll}
E^{ \pm} & 0 \\
F^{ \pm} & G
\end{array}\right)\left(\begin{array}{l}
x \\
y
\end{array}\right)+\left(\begin{array}{c}
0 \\
H
\end{array}\right)
$$

and $T=I^{ \pm} t$, where $I^{+} I^{-}>0, E^{+}, E^{-}>0$ and $G \neq 0$. So we have eight degrees of freedom and thus, for the problem of piecewise linear systems with two pieces we can reduce the initial twelve parameters to four in such a way that the piecewise linear differential system satisfies the conditions (i)-(iv). 
Moreover if we want also to force the existence of an isolated generalized singular point we must imply that $f^{+}\left(0, y_{0}\right)=f^{-}\left(0, y_{0}\right)=0$ for a convenient $y_{0}$, which in the case of piecewise linear systems means one parameter less.

\section{Proof of Theorem 1}

We start with the piecewise linear differential system (1) having the subsystems (4) and (5), and we use the 9 degrees of freedom coming from the twin affine changes of variables and by forcing that the point $(0,0)$ be an isolated generalized singular for fixing 9 of the parameters of the system. More precisely taking

$$
\begin{aligned}
& E^{+}=-\frac{\left(b^{-}+1\right) \sqrt{c^{+} b^{+}+b^{+}+c^{+}-a^{+} d^{+}+1}}{\left(b^{+}+1\right)\left(\alpha^{-}-1\right) \sqrt{c^{-} b^{-}+b^{-}+c^{-}-a^{-} d^{-}+1}}, \\
& E^{-}=\frac{1}{1-\alpha^{-}}, \\
& F^{+}=-\frac{a^{+}\left(b^{-}+1\right)}{\left(b^{+}+1\right)\left(\alpha^{-}-1\right) \sqrt{c^{-} b^{-}+b^{-}+c^{-}-a^{-} d^{-}+1}}, \\
& F^{-}=-\frac{a^{-}}{\left(\alpha^{-}-1\right) \sqrt{c^{-} b^{-}+b^{-}+c^{-}-a^{-} d^{-}+1}}, \\
& G^{-} \\
& H^{-}=-\frac{\left(b^{-}+1\right)\left(\alpha^{+} a^{+}+a^{+}+b^{+} \beta^{+}+\beta^{+}\right)}{\left(b^{+}+1\right)\left(\alpha^{-}-1\right) \sqrt{c^{-} b^{-}+b^{-}+c^{-}-a^{-} d^{-}+1}}, \\
& I^{+}=\sqrt{\left.c^{-}+1\right) \sqrt{c^{-} b^{-}+b^{-}+b^{-}+c^{-} d^{-}+1}}, \\
& I^{-}=\sqrt{c^{-} b^{-}+b^{-}+d^{+}+1},
\end{aligned}
$$

which are well defined if $0 \leq\left|a^{ \pm}\right|,\left|b^{ \pm}\right|,\left|c^{ \pm}\right|,\left|d^{ \pm}\right|,\left|\alpha^{ \pm}\right|,\left|\beta^{ \pm}\right|<<1$, and by forcing the existence of an isolated generalized singular point at (0,0), subsystems (4) and (5) become

$$
\left(\begin{array}{c}
\dot{x} \\
\dot{y}
\end{array}\right)=\left(\begin{array}{cc}
0 & 1 \\
1 & d_{1}
\end{array}\right)\left(\begin{array}{c}
x-1-\alpha \\
y
\end{array}\right)=\left(\begin{array}{c}
f^{+}(x, y) \\
g^{+}(x, y)
\end{array}\right)
$$

and

$$
\left(\begin{array}{c}
\dot{x} \\
\dot{y}
\end{array}\right)=\left(\begin{array}{cc}
0 & 1 \\
1 & d_{2}
\end{array}\right)\left(\begin{array}{c}
x+1 \\
y
\end{array}\right)=\left(\begin{array}{l}
f^{-}(x, y) \\
g^{-}(x, y)
\end{array}\right)
$$

where we have renamed as $d_{1}, d_{2}$ and $\alpha$ the parameters that now occupy the positions of the previous $d^{+}, d^{-}$and $\alpha^{+}$respectively. 
After tedious but easy computations the orbit passing through the point $(0, r)$ of the piecewise linear differential system (1) having the subsystems (6) and (7) is

$$
\begin{aligned}
\varphi^{+}(t, 0, r)= & \left(e ^ { - \frac { 1 } { 2 } ( d _ { 1 } + s _ { 1 } ) t } \left(2 s_{1} e^{\frac{1}{2}\left(d_{1}+s_{1}\right) t}(\alpha+1)+d_{1} e^{d_{1} t}\left(-1+e^{s_{1} t}\right)(\alpha+1)+\right.\right. \\
& e^{\left(d_{1}+s_{1}\right) t}\left(2 r-s_{1}(\alpha+1)\right)-e^{d_{1} t}\left(2 r+s_{1}(\alpha+1)\right), \\
& \left.\left.e^{\frac{1}{2}\left(d_{1}-s_{1}\right) t}\left(-d_{1} r+s_{1} r+e^{s_{1} t}\left(d_{1} r+s_{1} r-2 \alpha-2\right)+2 \alpha+2\right)\right) /\left(2 s_{1}\right)\right), \\
\varphi^{-}(t, 0, r)= & \left(e ^ { - \frac { 1 } { 2 } ( d _ { 2 } + s _ { 2 } ) t } \left(-d_{2} e^{d_{2} t}\left(-1+e^{s_{2} t}\right)-2 s_{2} e^{\frac{1}{2}\left(d_{2}+s_{2}\right) t}+e^{d_{2} t}\left(s_{2}-2 r\right)+\right.\right. \\
& e^{\left(d_{2}+s_{2}\right) t}\left(2 r+s_{2}\right), \\
& \left.\left.e^{\frac{1}{2}\left(d_{2}-s_{2}\right) t}\left(-d_{2} r+s_{2} r+e^{s_{2} t}\left(d_{2} r+s_{2} r+2\right)-2\right)\right) /\left(2 s_{2}\right)\right),
\end{aligned}
$$

where $s_{i}=\sqrt{d_{i}^{2}+4}$ for $i=1,2$.

We compute know the time $t^{ \pm}$depending on $r$ necessary for going from the point $(0, r)$ to the point $\left(0, r^{ \pm}\right)$through $x \geq 0$ for $t^{+}$, and through $x \leq 0$ for $t^{-}$. That is we move in positive time with the orbit $\varphi^{+}$from $(0, r)$ to $\left(0, r^{+}\right)$and in negative time with the orbit $\varphi^{-}$from $(0, r)$ to $\left(0, r^{-}\right)$. Since it is not possible to isolate $t$ from the first component of $\varphi^{ \pm}(t, 0, r)=\left(0, r^{ \pm}\right)$equalled to zero, we compute the Taylor series of them obtaining

$$
\begin{aligned}
t^{+}= & \frac{2 r}{\alpha+1}+\frac{2 d_{1} r^{2}}{3(\alpha+1)^{2}}+\frac{2\left(2 d_{1}^{2}+3\right) r^{3}}{9(\alpha+1)^{3}}+\frac{4 d_{1}\left(11 d_{1}^{2}+27\right) r^{4}}{135(\alpha+1)^{4}}+ \\
& \frac{2\left(52 d_{1}^{4}+180 d_{1}^{2}+81\right) r^{5}}{405(\alpha+1)^{5}}+\frac{2 d_{1}\left(60 d_{1}^{4}+268 d_{1}^{2}+243\right) r^{6}}{567(\alpha+1)^{6}}+ \\
& \frac{2\left(4\left(956 d_{1}^{4}+5229 d_{1}^{2}+7209\right) d_{1}^{2}+6075\right) r^{7}}{42525(\alpha+1)^{7}}+ \\
& \frac{8 d_{1}\left(356 d_{1}^{6}+2304 d_{1}^{4}+4284 d_{1}^{2}+2025\right) r^{8}}{18225(\alpha+1)^{8}}+O\left(r^{9}\right), \\
t^{-}= & -2 r+\frac{2 d_{2} r^{2}}{3}-\frac{2}{9}\left(2 d_{2}^{2}+3\right) r^{3}+\frac{4}{135} d_{2}\left(11 d_{2}^{2}+27\right) r^{4}- \\
& \frac{2}{405}\left(52 d_{2}^{4}+180 d_{2}^{2}+81\right) r^{5}+\frac{2}{567} d_{2}\left(60 d_{2}^{4}+268 d_{2}^{2}+243\right) r^{6}- \\
& \frac{2\left(4\left(956 d_{2}^{4}+5229 d_{2}^{2}+7209\right) d_{2}^{2}+6075\right) r^{7}}{42525}+ \\
& \frac{8 d_{2}\left(356 d_{2}^{6}+2304 d_{2}^{4}+4284 d_{2}^{2}+2025\right) r^{8}}{18225}+O\left(r^{9}\right),
\end{aligned}
$$

Now substituting $t=t^{ \pm}$in the second component of $\varphi^{ \pm}(t, 0, r)=\left(0, r^{ \pm}\right)$we can compute the Taylor expressions of the points $r^{ \pm}=R^{ \pm}(r)$. Both expressions start with linear terms in $r$ being the linear coefficient -1 in both cases. Then we can get the Poincaré return map $P\left(r^{-}\right)=R^{+}\left(\left(R^{-}\right)^{-1}\left(r^{-}\right)\right)$and compute the solutions of $P\left(r^{-}\right)=r^{-}$which will give the limit 
cycles. But since we have the expression of $R^{-}(r)$ already expressed in Taylor series and an inverse of it would have to be done in similar terms with an increasing complexity and loss of precision, instead of the usual Poincaré map we will consider $Q(r)=R^{+}(r)-R^{-}(r)=$ $r^{+}-r^{-}$, whose zeros determine exactly the same limit cycles. Then it is easy to determine $Q(r)$ which starts in quadratic terms on $r$ as:

$$
Q(r)=V_{2} r^{2}+V_{3} r^{3}+V_{4} r^{4}+V_{5} r^{5}+V_{6} r^{6}+V_{7} r^{7}+V_{8} r^{8}+\ldots,
$$

with

$$
\begin{aligned}
& V_{2}=2\left(d_{1}+d_{2}+d_{2} \alpha\right) /(3(\alpha+1)) \text {, } \\
& V_{3}=-4\left(-d_{1}+d_{2}+d_{2} \alpha\right)\left(d_{1}+d_{2}+d_{2} \alpha\right) /\left(9(\alpha+1)^{2}\right) \text {, } \\
& V_{4}=2\left(22 d_{1}^{3}+9 d_{1}+22 d_{2}^{3}+22 d_{2}^{3} \alpha^{3}+9 d_{2} \alpha^{3}+66 d_{2}^{3} \alpha^{2}+27 d_{2} \alpha^{2}+9 d_{2}+66 d_{2}^{3} \alpha+\right. \\
& \left.27 d_{2} \alpha\right) /\left(135(\alpha+1)^{3}\right) \text {, } \\
& V_{5}=-\left(4 \left(-26 d_{1}^{4}-27 d_{1}^{2}+26 d_{2}^{4}+26 d_{2}^{4} \alpha^{4}+27 d_{2}^{2} \alpha^{4}+104 d_{2}^{4} \alpha^{3}+108 d_{2}^{2} \alpha^{3}+27 d_{2}^{2}+\right.\right. \\
& \left.\left.156 d_{2}^{4} \alpha^{2}+162 d_{2}^{2} \alpha^{2}+104 d_{2}^{4} \alpha+108 d_{2}^{2} \alpha\right)\right) /\left(405(\alpha+1)^{4}\right), \\
& V_{6}=2\left(100 d_{1}^{5}+176 d_{1}^{3}+27 d_{1}+100 d_{2}^{5}+100 d_{2}^{5} \alpha^{5}+176 d_{2}^{3} \alpha^{5}+27 d_{2} \alpha^{5}+500 d_{2}^{5} \alpha^{4}+\right. \\
& 880 d_{2}^{3} \alpha^{4}+135 d_{2} \alpha^{4}+176 d_{2}^{3}+1000 d_{2}^{5} \alpha^{3}+1760 d_{2}^{3} \alpha^{3}+270 d_{2} \alpha^{3}+1000 d_{2}^{5} \alpha^{2}+ \\
& \left.1760 d_{2}^{3} \alpha^{2}+270 d_{2} \alpha^{2}+27 d_{2}+500 d_{2}^{5} \alpha+880 d_{2}^{3} \alpha+135 d_{2} \alpha\right) /\left(945(\alpha+1)^{5}\right) \text {, } \\
& V_{7}=-8\left(-956 d_{1}^{6}-2424 d_{1}^{4}-999 d_{1}^{2}+956 d_{2}^{6}+956 d_{2}^{6} \alpha^{6}+2424 d_{2}^{4} \alpha^{6}+999 d_{2}^{2} \alpha^{6}+\right. \\
& 5736 d_{2}^{6} \alpha^{5}+14544 d_{2}^{4} \alpha^{5}+5994 d_{2}^{2} \alpha^{5}+2424 d_{2}^{4}+14340 d_{2}^{6} \alpha^{4}+36360 d_{2}^{4} \alpha^{4}+ \\
& 14985 d_{2}^{2} \alpha^{4}+19120 d_{2}^{6} \alpha^{3}+48480 d_{2}^{4} \alpha^{3}+19980 d_{2}^{2} \alpha^{3}+999 d_{2}^{2}+14340 d_{2}^{6} \alpha^{2}+ \\
& \left.36360 d_{2}^{4} \alpha^{2}+14985 d_{2}^{2} \alpha^{2}+5736 d_{2}^{6} \alpha+14544 d_{2}^{4} \alpha+5994 d_{2}^{2} \alpha\right) /\left(42525(\alpha+1)^{6}\right), \\
& V_{8}=2\left(9968 d_{1}^{7}+33372 d_{1}^{5}+24372 d_{1}^{3}+2025 d_{1}+9968 d_{2}^{7}+9968 d_{2}^{7} \alpha^{7}+33372 d_{2}^{5} \alpha^{7}+\right. \\
& 24372 d_{2}^{3} \alpha^{7}+2025 d_{2} \alpha^{7}+69776 d_{2}^{7} \alpha^{6}+233604 d_{2}^{5} \alpha^{6}+170604 d_{2}^{3} \alpha^{6}+14175 d_{2} \alpha^{6}+ \\
& 33372 d_{2}^{5}+209328 d_{2}^{7} \alpha^{5}+700812 d_{2}^{5} \alpha^{5}+511812 d_{2}^{3} \alpha^{5}+42525 d_{2} \alpha^{5}+348880 d_{2}^{7} \alpha^{4}+ \\
& 1168020 d_{2}^{5} \alpha^{4}+853020 d_{2}^{3} \alpha^{4}+70875 d_{2} \alpha^{4}+24372 d_{2}^{3}+348880 d_{2}^{7} \alpha^{3}+1168020 d_{2}^{5} \alpha^{3}+ \\
& 853020 d_{2}^{3} \alpha^{3}+70875 d_{2} \alpha^{3}+209328 d_{2}^{7} \alpha^{2}+700812 d_{2}^{5} \alpha^{2}+511812 d_{2}^{3} \alpha^{2}+42525 d_{2} \alpha^{2}+ \\
& \left.2025 d_{2}+69776 d_{2}^{7} \alpha+233604 d_{2}^{5} \alpha+170604 d_{2}^{3} \alpha+14175 d_{2} \alpha\right) /\left(127575(\alpha+1)^{7}\right) \text {. }
\end{aligned}
$$

We note that since our Poincaré map $P$ is the composition of the two analytic Poincaré maps $R^{+}$and $\left(\left(R^{-}\right)^{-1}\right.$, it is analytic. So, in order to see that we have a center it is sufficient to show that all the constants $V_{i}$ 's are zero.

It is clear that $V_{2}=0$ implies $V_{3}=0$. Furthermore, when $V_{2}=0$ that is $d_{1}=-d_{2}(1+\alpha)$, 
we have

$$
\begin{aligned}
V_{4}= & 2 d_{2} \alpha(\alpha+2) /\left(15(\alpha+1)^{2}\right), \\
V_{5}= & -4 d_{2}^{2} \alpha(\alpha+2) /\left(15(\alpha+1)^{2}\right), \\
V_{6}= & 2 d_{2} \alpha(\alpha+2)\left(176 d_{2}^{2}(\alpha+1)^{2}+27\left(\alpha^{2}+2 \alpha+2\right)\right) 9 /\left(45(\alpha+1)^{4}\right), \\
V_{7}= & -8 d_{2}^{2} \alpha(\alpha+2)\left(808 d_{2}^{2}(\alpha+1)^{2}+333\left(\alpha^{2}+2 \alpha+2\right)\right) /\left(14175(\alpha+1)^{4}\right), \\
V_{8}= & 2 d_{2} \alpha(\alpha+2)\left(3708 d_{2}^{4}(\alpha+1)^{4}+2708 d_{2}^{2}\left(\alpha^{2}+2 \alpha+2\right)(\alpha+1)^{2}+\right. \\
& \left.225\left(\alpha^{4}+4 \alpha^{3}+7 \alpha^{2}+6 \alpha+3\right)\right) /\left(14175(\alpha+1)^{6}\right) .
\end{aligned}
$$

We see clearly that if $V_{2}=V_{4}=0$, then $V_{i}=0$ for $i=5,6,7,8$. But we want to check that all $V_{i}$ are zero for proving the existence of a center. So we take $d_{1}=-d_{2}(1+\alpha)$ and determine that in this case $V_{4}=2 d_{2} \alpha(\alpha+2) /\left(15(\alpha+1)^{2}\right)$. We separate the problem in two cases, either $d_{2}=0$ or $\alpha=0$ since the possibility $\alpha=-2$ would be large enough to destroy the structure of the system.

Case $d_{2}=0$. Then $d_{1}=0$ and we get that

$$
\begin{aligned}
& t^{+}=\log \left(\frac{-r-\alpha-1}{r-\alpha-1}\right), \\
& t^{-}=\log \left(\frac{1-r}{r+1}\right), \\
& r^{+}=-r \\
& r^{-}=-r
\end{aligned}
$$

which proves that the origin is a center.

Case $\alpha=0$. Then $d_{1}=-d_{2}$. In this case it is not possible to obtain an explicit expression of $t^{ \pm}$but it is easy to see that

$$
\begin{aligned}
& f^{+}(x, y)=f^{-}(x, y)=y, \\
& g^{+}(x, y)=-g^{-}(x, y)=-1+x-d_{2} y
\end{aligned}
$$

and consequently, by symmetry, the origin is a center.

In short we have that when $V_{2}=V_{4}=0$ there is a center at the origin. We can choose $\alpha$ and $d_{1}$ in such a way that $V_{4}=-\varepsilon$ and $V_{2}=\varepsilon^{2}$ with $\varepsilon>0$ a sufficiently small parameter. Then the center is broken becoming a stable focus a little far from the origin, but unstable very close to the origin. Consequently the origin has an infinitesimal stable limit cycle surrounding it for $\varepsilon>0$ a sufficiently small. More precisely, if we take $d_{2}<0$,

$$
\begin{aligned}
& \alpha=-\left(\frac{2 d_{1}\left(22 d_{1}^{2}+9\right)}{44 d_{2}^{3}+18 d_{2}-135 \varepsilon}\right)^{1 / 3}-1, \\
& d_{1}=\sqrt{-\frac{\left(3 \varepsilon^{2}+2 d_{2}\right)^{3}}{66 \varepsilon^{6}+88 d_{2}^{2} \varepsilon^{2}+60 \varepsilon+4 d_{2}\left(33 \varepsilon^{4}-2\right)}},
\end{aligned}
$$


then $V_{2}=\varepsilon^{2}$ and $V_{4}=-\varepsilon$. So, for $\varepsilon>0$ sufficiently small we have a stable limit cycle $\gamma$ surrounding the origin of coordinates.

Now if we perturb with terms of order $\varepsilon^{3}$ our discontinuous differential systems (6) and (7) as follows

$$
\left(\begin{array}{l}
\dot{x} \\
\dot{y}
\end{array}\right)=\left(\begin{array}{cc}
0 & 1 \\
1 & d_{1}
\end{array}\right)\left(\begin{array}{c}
x-1-\alpha \\
y
\end{array}\right)+\left(\begin{array}{c}
\varepsilon^{3} \\
0
\end{array}\right)
$$

and

$$
\left(\begin{array}{c}
\dot{x} \\
\dot{y}
\end{array}\right)=\left(\begin{array}{cc}
0 & 1 \\
1 & d_{2}
\end{array}\right)\left(\begin{array}{c}
x+1 \\
y
\end{array}\right)-\left(\begin{array}{c}
\varepsilon^{3} \\
0
\end{array}\right)
$$

with $\alpha$ and $d_{1}$ given by (9), we create for $\varepsilon>0$ sufficiently small the sliding limit cycle of Figure 4, inside the limit cycle $\gamma$. Obtaining in this way a second limit cycle around the origin. We note that the sliding segment of Figure 4 for our discontinuous differential system goes from the point $\left(-\varepsilon^{3}, 0\right)$ to the point $\left(\varepsilon^{3}, 0\right)$.

This completes the proof of Theorem 1 .

\section{Acknowledgments}

The first two authors are supported by MICIIN/FEDER grant MTM2008-03437 and AGAUR grant number 2009SGR-410. The second author is also supported by ICREA Academia. The last two authors are partially supported by CNPq and CAPES . All authors are also supported by the joint project CAPES-MECD grant PHB-2009-0025-PC and AUXPE-DGU 15/2010.

[1] A.A. Andronov, Les cycles limites de Poincaré et la théorie des oscillations autoentretenues, C. R. Acad. Sci. Paris 189 (1929), 559-561.

[2] A.A. Andronov, A.A. Vitt and S.E. Khaikin, Vibration Theory, Fizmatgiz, Moscow (in Russian, 1959

[3] N. N. BAutin, On the number of limit cycles which appear with the variation of the coefficients from an equilibrium position of focus or center type, Translations of the Amer. Math. Soc. 1, (1962) 396-413. Translated from russian paper of same title published by Math. USSR-Sb. (1954) 100, 397-413.

[4] M. di Bernardo, C.J. Budd, A.R. Champneys and P. Kowalcyk, PiecewiseSmooth Dynamical Systems, Theory and Applications, Springer-Verlag, London, 2008.

[5] S.N. Chow, C. Li And D. Wang, Normal Forms and Bifurcation of Planar Vector Fields, Cambridge Univ. Press., 1994.

[6] B. Coll, A. Gasull and R. Prohens, Degenerate Hopf bifurcation in discontinuous planar systems, J. Math. Anal. Appl. 253 (2001), 671-690. 
[7] Z. Du, Y. Li AND W. ZHAng, Bifurcation of periodic orbits in a class of Planar Filippov systems, Nonlinear Anal. Appl. 69(10) (2008), 3610-3628.

[8] F. Dumortier, J. Llibre, And J.C. Artés, Qualitative Theory of Planar Differential Systems, Universitext, Springer-Verlag, New York-Berlin, 2006.

[9] A.F. Filippov, Differential Equation with Discontinuous Right-Hand Sides, Kluver Academic, Netherlands, 1988.

[10] E. Freire, E. Ponce, F. Rodrigo And F. Torres, Bifurcation sets of continous piecewise linear systems with two zonesI, Int. J. Bifurcation and Chaos 11 (1998), 2073-2097.

[11] A. Gasull and J. Torregrosa, Center-focus problem for discontinuous planar differential equations, Inter. J. Bifur. Chaos Appl. Sci. Engrg. 13 (2003), 1755-1765.

[12] M. Kunze, Non-Smooth Dynamical Systems, Springer-Verlag, Berlin, 2000.

[13] T. Küpper And S. Moritz, Generalized Hopf bifurcation for non-smooth planar systems, Philos. Trans. R. Soc. Lond. Ser. A Math. Phys. Eng. Sci. 359 (2001), 24832496.

[14] Yu. A. Kuznetsov, S. Rinaldi and A. Gragnani, One-parameter bifurcations in planar Filippov systems, Inter. J. Bifur. Chaos Appl. Sci. Engrg. 8 (2003), 2157-2188.

[15] M. Han And W. Zhang, On Hopf bifurcation in non-smooth planar systems, J. of Diff. Eq. 248 (2010), 2399-2416.

[16] A. LiÉnARD, Etude des oscillations entretenues, Rev. Générale de l'Electricité 23 (1928), 901-912.

[17] X. LiU And M. Han, Hopf bifurcation for non-smooth Linéard systems, Inter. J. Bifur. Chaos Appl. Sci. Engrg. 19(7) (2009), 2401-2415.

[18] H. Poincaré, Mémoire sur les courbes définies par une équation differentielle I, II, J. Math. Pures Appl. 7 (1881), 375-422; 8 (1882), 251-96; Sur les courbes définies par les équation differentielles III, IV, 1 (1885), 167-244; 2 (1886), 155-217.

[19] VAN DER POL, On relaxation-oscillations, Phil. Mag. 2 (1926), 978-992.

[20] Ye Yanqian, Theory of Limit Cycles, Translations of Math. Monographs 66 (Providence, RI Amer. Math. Soc.), 1986.

[21] Y. Zou, T. KüpPer And W.J. Beyn, Generalized Hopf bifurcation for planar Filippov systems continuous at the origin, J. Nonlinear Sci. 16 (2006), 159-177. 